\section{Responsabilização pelo controle de resultados no Sistema Único de Saúde no Brasil}

\author{
Maria Arlete Duarte Araujo ${ }^{1}$
}

Como citar: Araujo MAD. Responsabilização pelo controle de resultados no Sistema Único de Saúde no Brasil. Rev Panam Salud Publica. 2010;27(3):230-6.

Palavras-chave: Indicadores de gestão; gestão em saúde; Sistema Único de Saúde; avaliação de resultados (cuidados de saúde); Brasil.

\footnotetext{
1 Correspondência: Avenida Rodrigues Alves 410/1100, Petrópolis, CEP 59020-200, Natal, RN, Brasil. E-mail: arletearaujo@natal.digi. com.br
}

A responsabilização pelo controle dos resultados da aplicação de políticas públicas realiza-se, em princípio, mediante avaliação a posteriori do desempenho dessas políticas e prestação de contas ao cidadão. Parte-se da premissa de que uma gestão orientada por resultados tem mecanismos de aferição do desempenho, da satisfação do usuário e de controle social, com incentivos vinculados a produtividade e eficiência que, articulados, configuram as condições necessárias para a responsabilização dos gestores públicos.

Esse tipo de responsabilização representa uma mudança radical na forma de prestar contas da ação pública à sociedade. Não se trata de uma prestação de contas vinculada apenas a aspectos de natureza processual e legal, mas de uma prestação de contas que contemple, também, aspectos de eficiência, eficácia e efetividade da ação pública. A idéia central dessa prestação de contas é a de que a responsabilização pelo controle de resultados, além de uma necessidade dos governantes para dar respostas à sociedade, atende às preocupações de minimizar o foco nos procedimentos e centrá-lo nos resultados (1-5).

Um conceito essencial na discussão da responsabilização pelo controle de resultados é a contratualização, uma vez que, através desse dispositivo, se estabelece o resultado desejado e, em função disso, torna-se possível estabelecer também as condições para o seu alcance (1). Um modelo contratual tem que incluir, necessariamente, metas, meios, controle e incentivos. Metas bem definidas servem para orientar a ação de gestão; os meios criam condições para o alcance das metas; o controle permite acompanhar os processos, a alocação de recursos, avaliar as dificuldades, enfim, avaliar a implementação das ações; e os incentivos constituem os elementos mobilizadores do comportamento humano em direção às metas. A vinculação entre metas, meios, controles e incentivos constituem, então, o cerne dos contratos de gestão (2). Assim, a contratualização deixa em segundo plano o controle burocrático e coloca em foco os resultados a serem obtidos. A obtenção de resultados é decorrente da pactuação entre as partes, que acordam entre si, além do desempenho esperado, os padrões de qualidade, o custo, a duração, as sistemáticas de avaliação e as formas de renovação do contrato.

Nessa perspectiva, fica evidente que o controle pelos resultados deve, antes de tudo, dar resposta a algumas perguntas. Para Behn (6), as questões que devem ser respondidas são: quem decide quais resultados deverão ser produzidos? Como o processo funciona? Quem pode ser cobrado (que é accountable) pela produção desses resultados? Quem responde pela implementação do processo de prestação de contas (pelo processo de accountability)? 
Outra perspectiva de análise é vincular a responsabilização a uma gestão pautada por indicadores de desempenho. Essa é a posição defendida por Yalta (7), quando afirma que uma gestão por resultados deve ter planos bem definidos, com missão e objetivos, cursos de ação e indicadores de desempenho e uma relação direta entre indicadores de desempenho e incentivos em nível institucional, grupal e individual. A ênfase nos indicadores de desempenho como requisito fundamental de uma gestão por resultados coloca um conjunto de decisões a serem tomadas para que eles possam ser utilizados, como a abrangência e as dimensões dos indicadores. Os indicadores permitem estabelecer um conjunto de controles sobre as atividades públicas sob óticas distintas, cobrindo assim um amplo leque de possibilidades de avaliação - custos, qualidade, economia, eficiência, produtividade, satisfação do usuário, gastos.

Uma outra vertente de análise vincula o tema da responsabilização à prática sistemática da avaliação enquanto mecanismo institucionalizado capaz de produzir não apenas mudanças de atitude em relação aos mecanismos de melhoria da ação governamental, mas também um debate político mais bem informado sobre as políticas e, consequentemente, um mecanismo capaz de produzir accountability $(8,9)$. A avaliação constitui, assim, um instrumento para a manutenção de uma cidadania informada acerca do desempenho do governo, o que pressupõe uma prestação de contas sistemática e uma clara identificação dos mecanismos de accountability (4).

Ainda outra forma de analisar a questão é considerar que a responsabilização pelo controle de resultados engloba tanto os aspectos de contratualização e de indicadores de desempenho como os de avaliação, ao situar-se no marco da gestão pública em que o resultado se converte na referência-chave quando aplicado a todo o processo de gestão. Assim, no caso da administração pública, a gestão por resultados tem os objetivos de: oferecer elementos de informação e intervenção aos responsáveis pela administração pública, contribuir para a melhoria da capacidade de prestar contas das organizações públicas, ajudar na alocação de objetivos e responsabilidades e possibilitar a avaliação de desempenho dos gestores públicos (3). Uma gestão em sintonia com os valores da prestação de contas é uma gestão que, além de se preocupar em criar uma estrutura de aferição de desempenho, é capaz de institucionalizar o acesso a essas informações e permitir que o cidadão se manifeste, seja em audiências públicas, através de pesquisas de opinião ou através de qualquer outro canal de participação.

Todos esses aspectos mostram que a tarefa de construção da responsabilização pelos resultados não é fácil. Talvez por isso, embora o discurso da responsabilização tenha ganho destaque nas iniciativas de reforma do Estado, ainda predomine a forma convencional de prestação de contas, em que se privilegiam os aspectos processuais e legais, deixando em segundo plano os aspectos vinculados a metas ou indicadores previamente pactuados entre os diversos atores no processo de formulação das políticas públicas.

Além disso, apenas aspectos pontuais da forma de responsabilização por controle de resultados vêm sendo estudados nos diversos âmbitos setoriais da administração pública, em especial no setor de saúde, que passou, a partir da década de 1990, por extensas reformas com o objetivo de implementar um sistema universal de atenção à saúde (10-13). Nesse sentido, o presente artigo tem por objetivo analisar, a partir do exame dos instrumentos normativos aprovados no sistema sanitário brasileiro após as reformas empreendidas, como se configura a responsabilização por controle de resultados. Dado que a reforma do sistema de saúde foi uma das mais importantes realizadas no marco da reforma do Estado no Brasil, é extremamente pertinente analisar se os instrumentos normativos asseguram formas de prestação de contas ao cidadão que não se limitem aos aspectos processuais e legais, traços característicos do modelo burocrático de administração pública que a reforma do Estado pretende alterar mediante a ampliação das formas de controle.

\section{CARACTERIZAÇÃO DO SISTEMA ÚNICO DE SAÚDE}

O Sistema Único de Saúde é constituído pelo conjunto de ações e serviços de saúde prestados por órgãos e instituições públicas federais, estaduais e municipais com base nos princípios de universalidade, equidade e integralidade (14). A direção única do SUS é exercida em cada esfera de governo pelos seguintes órgãos: no âmbito da União, pelo Ministério da Saúde; no âmbito dos estados e do Distrito Federal, pelas secretarias estaduais de saúde ou órgão equivalente; no âmbito dos municípios, pelas secretaria municipais de saúde ou órgão equivalente. O SUS conta ainda, em cada esfera de governo, com as seguintes instâncias colegiadas: a Conferência de Saúde e o Conselho de Saúde (15). A Conferência de Saúde se reúne a cada 4 anos com a representação dos vários segmentos sociais para avaliar a situação de saúde e propor diretrizes para a formulação da política de saúde nos níveis correspondentes, enquanto que o Conselho de Saúde congrega representantes do governo, prestadores de serviço, profissionais de saúde e usuários e atua na formulação de estratégias e no controle da execução da política de saúde, inclusive nos aspectos econômicos e financeiros. Participam do Conselho Nacional de Saúde um Conselho Nacional de Secretários de Saúde (Conass) e um Conselho Nacional de Secretários Municipais de Saúde (Conasems).

O SUS é caracterizado pela universalidade de cobertura, processo de descentralização político-administrativo que envolve a transferência de serviços, responsabilidades e recursos da esfera federal para as esferas estadual e municipal, integração de todos os serviços públicos de saúde, gestão apoiada nas relações intergestores, participação da iniciativa privada como provedor do sistema de saúde, controle social 
como elemento estruturante do sistema de saúde e democratização e incorporação de novos atores sociais ao sistema de saúde. A operacionalização do SUS tem sido feita através de um conjunto de normas operacionais, que são diretrizes do governo federal para orientar o funcionamento do sistema (15-33).

\section{RESPONSABILIZAÇÃO PELO CONTROLE DE RESULTADOS NO SUS}

No presente artigo, buscou-se nos instrumentos normativos um conjunto de categorias vinculadas à forma de responsabilização pelo controle de resultados, definidas a partir da literatura que aborda a Nova Gestão Pública como paradigma dominante na discussão sobre a reforma do Estado (4, 6, 34). Assim, buscou-se verificar se as normas estabelecem indicadores de desempenho na gestão, avaliação ex post (realizada após a execução das ações com o objetivo de verificar o cumprimento das metas previamente definidas), pesquisas de satisfação do usuário com a prestação dos serviços de saúde e mecanismos de controle social. O pressuposto é que esses elementos, se forem articulados corretamente, podem criar as condições necessárias para produzir uma mudança de orientação na gestão pública, invertendo o foco dos procedimentos para os resultados.

Discutir a responsabilização pelo controle de resultados na área da saúde pública no Brasil implica discutir as condições de gestão que foram criadas para o conjunto das ações e serviços de saúde a partir da lei $8080 / 90$ (14). São muitas as formas de apreender as condições de gestão e variados os seus aspectos, mas nos interessa nesta análise examinar os mecanismos gerenciais voltados para criar uma cultura gerencial orientada pelos resultados.

\section{Indicadores}

Em primeiro lugar, sem prioridade na ordem da discussão, há um conjunto de dispositivos normativos que incentivam o uso de indicadores de desempenho como uma ferramenta gerencial para avaliar o resultado da gestão a partir de 1998. A primeira iniciativa foi a portaria 3 925/98 (26) que, ao aprovar o Manual para a Organização da Atenção Básica no SUS, propôs um conjunto de indicadores como instrumento para acompanhamento e avaliação dos resultados obtidos através da implementação das ações relativas à atenção básica (entre outros, percentual de crianças menores de 4 meses com aleitamento materno exclusivo, percentual de internações hospitalares de crianças menores de 1 ano residentes no município, cobertura vacinal de rotina de crianças menores de 1 ano, número de hospitalizações por tétano neonatal, por local de residência, número de hospitalizações por difteria, por local de residência, incidência de casos de sarampo confirmados, número de hospitalizações por coqueluche, por local de residência, número de hospitalizações por tétano acidental, por local de residência, prevalência de desnutrição em menores de 5 anos, por local de residência, prevalência de desnutrição em maiores de 60 anos, por local de residência).

Em 2001, a portaria 95 NOAS/SUS (19) procurou consolidar o uso dos indicadores de desempenho, colocando-os como exigência para que os municípios habilitados nas condições de gestão da NOB/SUS $01 / 96$ (18) pudessem receber o Piso de Atenção Básica Ampliado, e incluindo-os como parte integrante do Plano Municipal de Saúde (Quadro de Metas). Além do estímulo para que os gestores passassem a utilizar os indicadores no planejamento e execução de suas ações, a portaria 95/01 (19) também trouxe a definição de áreas de atuação estratégicas mínimas para os municípios habilitados na condição de Gestão Plena da Atenção Básica Ampliada. Essa definição de áreas de atuação objetivava fazer com que a gestão do município tivesse foco e pudesse ser avaliada a partir do uso de indicadores. A portaria 95/01 (19) inovou também ao tentar definir remuneração por realização de metas, estabelecendo que unidades hospitalares públicas sob gerência de um nível de governo e gestão de outro, habilitado em gestão plena do sistema, preferencialmente deixassem de ser remunerados por produção de serviços e passassem a receber recursos correspondentes à realização de metas estabelecidas de comum acordo.

Novos instrumentos de gestão foram criados com a portaria 548/01 (27): agendas, planos de saúde, quadros de metas e relatórios de gestão. Todos foram balizados pelo princípio de definição de indicadores de saúde. Nessa linha, as agendas nacional, estaduais e municipais de saúde devem destacar e justificar os eixos prioritários de intervenção, os objetivos, os indicadores e as metas prioritárias da política de saúde em cada esfera de governo e em cada exercício anual; os quadros nacional, estaduais e municipais de metas, parte integrante dos planos de saúde, deverão conter os indicadores e as metas prioritárias de cada esfera de governo em cada exercício anual. Em 2002, a portaria 373/02 NOAS/SUS 01/02 (20) ratificou as áreas estratégicas de atuação mínimas da condição de Gestão Plena da Atenção Básica Ampliada do município. No entanto, foi com a portaria 21/05 (21) que se aprovou a relação de indicadores da atenção básica que devem ser pactuados entre municípios, estados e Ministério da Saúde (entre esses indicadores, número absoluto de óbitos em menores de 1 ano de idade; taxa de mortalidade infantil; proporção de nascidos vivos com baixo peso ao nascer; proporção de óbitos em menores de 1 ano por causas mal definidas; taxa de internações por infecção respiratória aguda em menores de 5 anos de idade; homogeneidade da cobertura vacinal por tetravalente em menores de 1 ano de idade; taxa de mortalidade materna; proporção de nascidos vivos de mães com quatro ou mais consultas de pré-natal; proporção de óbitos de mulheres em idade fértil investigados; razão entre exames citopatológicos cérvico-vaginais em mulheres de 25 a 59 anos e a população feminina nessa faixa etária; taxa de internações por acidente 
vascular cerebral; taxa de mortalidade por doenças cérebro-vasculares).

A portaria 21/05 (21) não só definiu os indicadores mas também a sua forma de cálculo, e orientou acerca de como eles devem ser analisados. Em 2006, a portaria 493 (22) aprovou a relação de indicadores da atenção básica e, para reforçar a sua implementação, estabeleceu mecanismos, fluxos e prazos para o processo de pactuação das metas entre os gestores das três esferas de governo.

Um novo instrumento gerencial foi criado com a portaria 699/06 (23), que regulamenta as diretrizes operacionais dos Pactos pela Vida e de Gestão para consolidar o processo de pactuação dos indicadores entre os gestores e estabelecer as responsabilidades relativas a metas e objetivos do Pacto pela Vida. Trata-se dos termos de compromisso de Gestão Municipal, Estadual, do Distrito Federal e Federal, que tornam públicos os indicadores de monitoramento.

Dentro de uma lógica racionalizadora, a portaria 91/07 (25) unificou o processo de pactuação de indicadores e estabeleceu os indicadores do Pacto pela Saúde. O Ministério da Saúde define, para cada unidade da federação, as metas a serem alcançadas. Os estados, por sua vez, promovem a pactuação das metas com os municípios. Este esforço de pactuação é de fato inovador na gestão do SUS, na medida em que as três esferas de governo têm a oportunidade de discutir as prioridades e as metas a serem perseguidas, induzindo uma gestão orientada para resultados e favorecendo a transparência e os mecanismos de controle social. Além de inovador, é uma prática gerencial capaz de minimizar o forte grau de improvisação na gestão para o tratamento das questões relativas à saúde. Outrossim, uma gestão balizada por indicadores e metas cria condições efetivas de avaliação pelos cidadãos. Se os cidadãos não conhecem as metas a serem perseguidas, e se não há indicadores para aferir desempenho, como pode o sistema ser accountable?

\section{Avaliação ex-post}

Um outro aspecto da gestão diretamente relacionado ao uso de indicadores, a definição de metas, a elaboração de planos e relatórios de gestão, é a prática da avaliação ex-post, que significa a comparação de metas estabelecidas com as metas realizadas. A avaliação é a base da prestação de contas, pois permite verificar o acerto e os erros da tomada de decisão em um momento passado, ao mesmo tempo em que permite pensar o futuro.

No início do SUS, a avaliação contemplava apenas aspectos de natureza legal. A lei 8 080/90 (14) dizia apenas que o Ministério da Saúde acompanharia, através de seu sistema de auditoria, a conformidade à programação aprovada da aplicação dos recursos repassados a Estados e Municípios. Com a aprovação da NOB/SUS 01/91 (16), manteve-se a função fiscalizadora do Ministério da Saúde e introduziu-se o relatório de gestão como requisito básico para a trans- ferência automática de recursos aos municípios. A obrigatoriedade do relatório, que deveria conter o plano de trabalho elaborado, plano de trabalho executado e resultados alcançados, representava um esforço de comparação entre aquilo que fora estabelecido com o que fora realizado.

Em 1994, o decreto 1 232/94 (28) reafirmou a função de controle do Ministério da Saúde sobre a conformidade da aplicação dos recursos transferidos à programação dos serviços e ações constantes dos planos de saúde. Complementando o esforço de avaliação, o decreto 1 651/95 (29) regulamentou o Sistema Nacional de Auditoria. Manteve-se o foco nos procedimentos, mas houve uma abertura para avaliação dos resultados mediante prestação de contas e relatório de gestão, que deve conter a programação e a execução física e financeira do orçamento, do projeto, dos planos e das atividades e a comprovação dos resultados alcançados quanto à execução do plano de saúde.

A NOB/SUS 1/96 (18), em função do processo de reorientação do modelo de atenção, defendeu o aperfeiçoamento e a disseminação dos instrumentos e técnicas de avaliação de resultados e do impacto das ações do SUS. Estabeleceu ainda que a avaliação do cumprimento das ações programadas em cada nível de governo deve ser feita em relatório de gestão anual. A portaria 3 925/98 (26), de forma complementar à NOB/SUS 1/96 (18), propôs, como instrumento para acompanhamento e avaliação dos resultados obtidos das ações relativas à atenção básica, um conjunto de indicadores. A preocupação residiu em criar mecanismos que permitam avaliar de forma objetiva o impacto das ações sobre as condições de saúde da população.

Novas complementações para tornar consolidado o uso dos indicadores vieram com a portaria 476/99 (30), que determinou que o desempenho dos indicadores deve ser avaliado ao final de cada exercício e que as metas dos indicadores pactuados deve fazer parte do Plano Estadual de Saúde. Com a compreensão de que o fortalecimento das funções de controle e avaliação dos gestores é fundamental no processo de construção do SUS, e de que apenas o uso dos indicadores era insuficiente, a portaria 95/01 (19) orientou que a avaliação a ser realizada pelas três esferas de governo deveria priorizar as seguintes dimensões: avaliação da organização do sistema e do modelo de gestão; relação com os prestadores de serviços; qualidade da assistência e satisfação dos usuários; resultados e impacto sobre a saúde da população, tendo como instrumento básico para o acompanhamento e avaliação dos sistemas de saúde o relatório de gestão.

Vinculada à preocupação de produzir resultados, a portaria 699/06 (23), que regulamenta as diretrizes operacionais dos Pactos Pela Vida e de Gestão, normatiza que o Termo de Cooperação entre Entes Públicos deve conter as metas e um plano operativo do acordo; as unidades públicas prestadoras de serviço devem, preferencialmente, receber os recursos de cus- 
teio correspondentes à realização das metas pactuadas no plano operativo e não por produção; e o processo de monitoramento do Pacto deverá ser orientado pelos indicadores, objetivos, metas e responsabilidades que compõem o respectivo Termo de Compromisso de Gestão.

Avaliando-se o conjunto de dispositivos normativos, fica claro qual é o modelo de gestão que se quer implantar: um modelo que priorize resultados, expressos através da melhoria dos indicadores de saúde; que se utilize de todos os instrumentos de gestão agenda, planos, quadros de metas e relatórios de gestão; que valorize as funções de controle e avaliação, de modo que elas apontem os problemas a corrigir e os acertos das decisões e, portanto, aquilo que deve ser mantido ou aperfeiçoado. Também fica claro, pelas sucessivas normas que estão sempre a reafirmar determinados procedimentos, que o sistema se move muito lentamente na direção desejada, exigindo às vezes que muitos mecanismos de indução sejam criados. Este fato evidencia, de um lado, a importância das normas no desenho e arquitetura do modelo, e do outro, a dificuldade de criar uma capacidade institucional de gestão apenas com as normas.

\section{Satisfação do usuário e controle social}

É visível também que a legislação estruturante do SUS é ampla na perspectiva do controle social. A ação fiscalizadora dos Conselhos se expressa de várias formas. A resolução 258/91 (16) define que os Conselhos de Saúde atuarão na formulação de estratégias e no controle da execução da política de saúde na instância correspondente, inclusive nos aspectos econômicos e financeiros, e que aprovarão os planos de saúde em cada esfera de sua atuação, bem como a fiscalização da movimentação dos recursos repassados às secretarias estaduais e municipais e/ou fundos de saúde.

A portaria 393/01 (31), que institui a Agenda de Saúde, define que as agendas estaduais e municipais serão aprovadas pelos respectivos Conselhos de Saúde. Por sua vez, a portaria 548/01 (27) reafirmou o poder dos Conselhos ao normatizar que as Agendas de Saúde, ao serem consolidadas e adaptadas em cada esfera de governo, comporão um processo de responsabilização progressiva, tendo por base as referências políticas, epidemiológicas e institucionais de cada esfera, sempre com a homologação do Conselho de Saúde correspondente, e que os Planos de Saúde, que são documentos de intenções políticas, de diagnóstico, de estratégias, de prioridades e de metas, vistos sob uma ótica analítica, devem ser submetidos na íntegra aos Conselhos de Saúde correspondentes, em cada nível de gestão do SUS.

A portaria 699/06 (23) por sua vez, definiu que os Termos de Compromisso da gestão municipal, estadual, do Distrito Federal e da União deverão ser aprovados pelos Conselhos municipais, estaduais, do Distrito Federal e da União, respectivamente. A portaria 3 277/06 (32), ao dispor sobre a participação comple- mentar dos serviços privados de assistência à saúde no âmbito do SUS, disciplinou que a necessidade de complementação de serviços deverá ser aprovada pelo Conselho de Saúde e constar do Plano de Saúde, e que as metas serão definidas pelo gestor em conjunto com o prestador, de acordo com as necessidades e peculiaridades da rede de serviços, devendo ser submetidas ao Conselho de Saúde.

Em 2007, a portaria 91 (25), ao unificar o processo de pactuação de indicadores, normatizou que as metas pactuadas pelos municípios, estados, Distrito Federal e União deverão passar por aprovação dos respectivos Conselho de Saúde. Novamente, é importante destacar o papel dos Conselhos, dado que as metas pactuadas orientarão todo o processo de gestão e refletem próprio quadro de saúde populacional que se deseja alcançar. Ainda em 2007, ao se regulamentar o financiamento e a transferência dos recursos federais para as ações e serviços de saúde, a portaria 204/07 (33) normatizou que a comprovação da aplicação dos recursos repassados pelo Fundo Nacional de Saúde aos fundos de saúde dos estados, do Distrito Federal e dos municípios, far-se-á para o Ministério da Saúde, mediante relatório de gestão, que deve ser elaborado anualmente e aprovado pelo respectivo Conselho de Saúde.

Constata-se que a ação fiscalizatória que os Conselhos poderão exercer é enorme. A ação fiscalizatória, vista pela possibilidade da aprovação de procedimentos/projetos/propostas, contempla desde autorização para guias de internamento, planejamento das atividades ambulatoriais, termos de compromisso dos gestores, a compra de serviços à iniciativa privada, relatórios de gestão, até projetos que o município deseja implementar. É claro que a simples atribuição de competências por si só não é suficiente para uma atuação efetiva dos Conselhos. Já há bastante discussão acumulada nesse sentido. No entanto, o fato de que toda a legislação reforça de forma contínua o poder dos Conselhos abre a possibilidade real de que condições possam ser criadas para tornar efetiva essa fiscalização.

Um outro aspecto relacionado à capacidade de fiscalização do cidadão são os dispositivos voltados para identificar a satisfação do usuário com a prestação de serviços. Nesse sentido, a legislação deixa muito a desejar, pois em apenas duas portarias constata-se a preocupação com a satisfação do usuário. São as portarias 95 (NOAS/SUS 01/01) (19) e 373 (NOAS/SUS 01/02) (20), que recomendam que a avaliação da qualidade da atenção pelos gestores deve envolver tanto a implementação de indicadores objetivos baseados em critérios técnicos como a adoção de instrumentos de avaliação da satisfação dos usuários do sistema que considerem a acessibilidade, a integralidade da atenção, a resolubilidade e a qualidade dos serviços prestados. Atente-se para o fato de que é uma recomendação. Além dessas portarias, o que há é um imenso vazio de quase 20 anos, revelando que a aposta do SUS está centrada na ação dos Conselhos, pelo fato já apontado de que a legislação lhes confere grande 
poder. Deixa-se, portanto, de contar com a satisfação do usuário como mais um recurso que pode melhorar a gestão e contribuir para tornar o sistema accountable ao cidadão.

\section{Conclusões}

É claro que a melhoria da capacidade institucional de gestão não ocorre pela simples existência dos instrumentos normativos, dado que a gestão envolve um conjunto de conhecimentos, habilidades e competências vinculados às distintas áreas funcionais da administração que extrapolam o campo normativo. Porém, as normas têm um papel importante na indução de práticas e comportamentos gerenciais, em especial pelo caráter coercitivo que apresentam, em maior ou menor grau.

A presente análise de um conjunto de instrumentos normativos evidencia que, em relação à responsabilização pelo controle de resultados, estão presentes os seguintes elementos: esforço de construção de uma gestão orientada para resultados, adoção de muitas ferramentas gerenciais (planos, relatórios, agendas, indicadores, pactuação de metas), esforço para que o sistema de saúde adote os indicadores de desempenho como uma ferramenta de gestão e avaliação vista como uma ferramenta imprescindível da gestão. A análise também revelou que o esforço para transformar uma gestão pública burocrática orientada por processos para uma gestão pública orientada para resultados apresenta dificuldades, tais como:

- o cidadão é um ator ausente da construção do sistema. Essa ausência é determinada por um desenho institucional que se limita a dar espaço aos Conselhos de Saúde, mas que não articula outros espaços de escuta ao cidadão;

- os mecanismos de prestação de contas se limitam a aspectos burocráticos próprios da administração pública, seguindo o caminho tradicional de prestação de contas aos órgãos de controladoria e auditoria. Isso significa dizer que a gestão ainda não é avaliada pelos resultados que ela alcança;

- os usuários não participam da tomada de decisão sobre as políticas de saúde e sobre os arranjos operacionais, o que implica que a gestão do SUS não tem se preocupado com a ampliação do espaço público do cidadão; e

- a eficácia e a efetividade dos contratos não são avaliadas, o que resulta em uma gestão pouco capaz de atender aos requisitos de uma gestão orientada por resultados.

Portanto, é possível afirmar apenas que no Brasil estão sendo criadas, de forma muito lenta, as condições para que essa forma de responsabilização se materialize. Ainda há muito a fazer para a consolidação de uma capacidade institucional de gestão com foco em resultados. Além das dificuldades já citadas, pode-se acrescentar a necessidade de reduzir as dispa- ridades referentes à competência de gestão, dada a heterogeneidade dos municípios brasileiros, de lidar com a falta de investimentos sistemáticos na capacitação dos recursos humanos, de fazer com que os Conselhos de Saúde atuem de forma efetiva no fiel cumprimento das normas e de ampliar novos espaços de escuta do cidadão, com a implantação das ouvidorias e demais fóruns de deliberação pública. É importante também apontar como um fator limitante o fato de que não se percebe que a prestação de contas ao cidadão seja um valor perseguido nos instrumentos normativos. Assim, não se vislumbra a forma de responsabilização pelo controle de resultados no SUS, apesar das importantes iniciativas já tomadas para implementar uma gestão orientada pelos resultados.

Agradecimentos. O projeto ao qual este artigo está vinculado, "Responsabilização na Reforma do Sistema de Saúde Catalunha e Brasil", teve apoio da Coordenação de Aperfeiçoamento de Pessoal de Nível Superior (CAPES).

\section{SYNOPSIS}

\section{Results-oriented management and accountability in the Brazilian Unified Health System}

By examining normative instruments, the present article evaluates if the discourse of results-oriented management and accountability has become a reality within the Brazilian health care system following the reform started in the 1990s with the establishment of the Unified Health System (SUS). The analysis shows that there is, in fact, an effort aimed at the construction of results-oriented management, with the incorporation of many management tools (plans, reports, agendas, indicators, joint goals-setting). Also, the health care system has strived to adopt performance indicators and views evaluation as an essential management tool. However, difficulties are also evident, such as the absence of citizens in the construction of the system, due to an institutional design that only provides space for official bodies (Health Councils); accountability mechanisms that are limited to bureaucratic aspects typical of public administration, following the traditional accountability path of audit and controllership; no engagement of health care system users in the decisionmaking about health care policies and operating arrangements; and no evaluation of the efficacy and effectiveness of contracts, placing the current management systems far from results-oriented management. It is possible to state that the conditions for accountability are slowly being created; however, it is still not possible to foresee the consolidation of resultsoriented management and accountability within the SUS.

Key words: Management indicators; health management; Unified Health System; outcome assessment (health care); Brazil. 


\section{REFERÊNCIAS}

1. Martins HF. Cultura de resultados e avaliação institucional: avaliando experiências contratuais na administração pública federal. Rev Eletronica Sobre a Reforma do Estado. 2007;8(jan/fev). Disponível em: direitodoestado.com/ revista / RERE-8-DEZEMBRO-2006HUMBERTO\%20MARTINS.pdf. Acessado em 23 de setembro de 2008.

2. Martins HF. Gestão de recursos públicos: orientação para resultados e accountability. Rev Eletronica Sobre a Reforma do Estado. 2005; 3(set/out/Nov). Disponível em: http://www.direito doestado.com.br/rere/edicao/03/. Acessado em 5 de março de 2010.

3. Serra A. Modelo aberto de gestão para resultados no setor público. Natal: SEARH/RN; 2008.

4. Quirós MM. Responsabilização pelo controle de resultados. Em: Bresser Pereira LC, Grau NC, Gorisman E. Responsabilização na administração pública. São Paulo: CLAD/Fundap; 2006. Pp. 165216.

5. Trosa S. Gestão pública por resultados: quando o Estado se compromete. Rio de Janeiro: Revan; 2001.

6. Behn RD. O novo paradigma da gestão pública e a busca da accountability democrática. Rev Serv Publ. 1998;49(4): $5-45$.

7. Yalta NS. VII Congreso Internacional del CLAD sobre la Reforma del Estado y de la Administración Pública. Lisboa, 8-11 de outubro de 2002. Disponível em: unpan1.un.org/intradoc/groups/ public/documents/CLAD/clad0043 703.pdf. Acessado em 5 de março de 2010.

8. Vaitsman J, Paes-Sousa R. Avaliação e gestão democrática de políticas públicas. IX Congresso Internacional da Brazilian Studies Association, (BRASA). Louisiana, 27 a 29 de março de 2008

9. Ospina S. Evaluación de la gestión pública: conceptos y aplicaciones en el caso latinoamericano. V Congreso Internacional del CLAD sobre la Reforma del Estado y de la Administración Pública. Santo Domingo, 24 a 27 de outubro de 2000. Disponível em: unpan1.un.org/ intradoc/groups/public/documents/C LAD/clad0038514.pdf. Acessado em 13 de janeiro de 2010.

10. El-Warrak LO. A contratualizaação no âmbito da gestão pública em saúde: a experiência da área de planejamento 4 do município do Rio de Janeiro [dissertação]. Rio de Janeiro: Fundação Oswaldo Cruz; 2008.

11. Pompeu JC. A situação contratual da rede hospitalar privada vinculada ao SUS: alguns elementos para reflexão [dissertação]. Rio de Janeiro: Escola Nacional de Saúde Pública/Fundação Oswaldo Cruz; 2004.

12. Ungarette MR. Contrato de gestão. Uma das formas de controle das agências reguladoras pelo governo: o caso da ANS [dissertação]. Rio de Janeiro: Fundação Oswaldo Cruz; 2004.

13. Peci A, Figale J, Oliveira F, Barragat A, Souza C. Oscips e termos de parceria com a sociedade civil: um olhar sobre o modelo de gestão por resultados do governo de Minas Gerais. Rev Adm Publica. 2008;42(6):1137-62.

14. Brasil, Ministério da Saúde. Lei 8 080/1990. Brasília: Ministério da Saúde. Disponível em: promenino.org.br/ Ferramentas/Conteudo/tabid/77/ ConteudoId/6def1b53-972d-4b26-9f38bf7b78ee1ea2/Default.aspx. Acessado em 4 de março de 2010.

15. Brasil, Casa Civil. Lei 8 142/1990. Disponível em: planalto.gov.br/CCIVIL/ leis/L8142.htm. Acessado em 4 de março de 2010.

16. Brasil, Instituto Nacional de Assistência Médica da Previdência Social (INAMPS). Resolução 258/1991. Disponível em: siops.datasus.gov.br/legislacao.php?esc= 4. Acessado em 4 de março de 2010.

17. Brasil, Ministério da Saúde. Portaria 545/1993. Brasília: Ministério da Saúde. Disponível em: siops.datasus.gov.br/ Documentacao/Portaria\%20545_20 05_1993. pdf. Acessado em 4 de março de 2010.

18. Brasil, Ministério da Saúde. Portaria 2 203/1996. Brasília: Ministério da Saúde. Disponível em: www.portalsocial.ufsc. br/legislacao/saude/nob96.pdf. Acessado em 4 de março de 2010

19. Brasil, Ministério da Saúde. Portaria 95/GM/2001. Brasília: Ministério da Saúde. Disponível em: siops.datasus. gov.br/Documentacao/Noas $\% 2001 \% 2$ 0de\%202001.pdf. Acessado em 4 de março de 2010.

20. Brasil, Ministério da Saúde. Portaria 373/2002. Brasília: Ministério da Saúde. Disponível em: dtr2004.saude.gov.br/ dab/docs/legislacao/noas_2002.pdf. Acessado em 4 de março de 2010.

21. Brasil, Ministério da Saúde. Portaria 21/ 2005. Brasília: Ministério da Saúde. Disponível em: dtr2001.saude.gov.br/sas/ PORTARIAS / P ort 2005 / GM / GM-21. htm. Acessado em 4 de março de 2010.

22. Brasil, Ministério da Saúde. Portaria 493/GM/2006. Brasília: Ministério da Saúde. Disponível em: saude.mg.gov. br/atos normativos/legislacaosanitaria/estabelecimentosde-saude/atencao-basica/portaria 493. pdf. Acessado em 4 de março de 2010.

23. Brasil, Ministério da Saúde. Portaria 699/GM, de 30 de março de 2006. Brasília: Ministério da Saúde. Disponível em: portal.saude.gov.br/portal/ arquivos/pdf/Portaria_699_2006.pdf. Acessado em 4 de março de 2010.

24. Brasil, Ministério da Saúde. Portaria 1 097/2006. Brasília: Ministério da Saúde. Disponível em: dtr2001.saude.gov.br/ sas/cpa/PortariaGM1097.pdf. Acessado em 5 de março de 2010 .
25. Brasil, Ministério da Saúde. Portaria 91/GM/2007. Brasília: Ministério da Saúde. Disponível em: dtr2004.saude. gov.br/dab/docs/legislacao/por taria91_10_01_07.pdf. Acessado em 5 de março de 2010.

26. Brasil, Ministério da Saúde. Portaria 3 925/1998. Disponível em: www.google. com.br/search?q=Portaria $+3925 \% 2$ F19 $98+$ aprova + o+manual\&btnG=Pesquisa r\&hl=pt-BR\&rls=com.microsoft $\% 3 \mathrm{Apt}-$ br\%3AIE-SearchBox\&rlz=1I7ADBR_pt$B R \& s a=2$. Acessado em 5 de março de 2010.

27. Brasil, Ministério da Saúde. Portaria 548/2001. Brasília: Ministério da Saúde. Disponível em: portal.saude.gov.br/ portal/arquivos/pdf / Portaria\% 20548.pdf. Acessado em 5 de março de 2010.

28. Brasil. Decreto 1 232/1994. Disponível em: http://www.jusbrasil.com.br/ legislacao/112869/decreto-1232-94. Acessado em 5 de março de 2010.

29. Brasil. Decreto 1 651/1995. Disponível em: dtr2004.saude.gov.br/susdeaz/ legislacao/arquivo/17_Decreto_1651. pdf. Acessado em 5 de março de 2010.

30. Brasil, Ministério da Saúde. Portaria 476/1999. Brasília: Ministério da Saúde. Disponível em: sna.saude.gov.br/legisla/legisla/gest/. Acessado em 5 de março de 2010.

31. Brasil, Ministério da Saúde. Portaria 393/2001. Brasília: Ministério da Saúde. Disponível em: bvsms.saude.gov.br/ bvs/saudelegis/gm/2001/prt0393_29 03_2001.html. Acessado em 5 de março de 2010.

32. Brasil, Ministério da Saúde. Portaria 3 277/2006. Brasília: Ministério da Saúde. Disponível em: www.mp.ba.gov.br/ atuacao/cidadania/gesau/legislacao/ temas/participacao.asp. Acessado em fevereiro de 2010.

33. Brasil, Ministério da Saúde. Portaria 204/GM/2007. Brasília: Ministério da Saúde. Disponível em: dtr2001.saude. gov.br/sas/PORTARIAS/Port2007/ GM/GM-204.htm. Acessado em 5 de março de 2010.

34. Casasnovas GL, Puig-Junoy J, Ganuza JJ, Miret IP. Los nuevos instrumentos de la gestión pública. Barcelona: Caja de Ahorros y Pensiones de Barcelona; 2003. (Colección Estudios Económicos 31). Disponível em: www.lacaixa.comunicacions.com/se/pbei.php?idioma=esp\&ll ibre $=31$. Acessado em 5 de março de 2010.

Manuscrito recebido em 28 de junho de 2009. Aceito em versão revisada em 14 de outubro de 2009. 